\title{
Gesellschaftlicher und rechtlicher Status der Sprach- meister*innen in der Frühen Neuzeit. Sprachliche Fähigkeiten als entscheidendes Kriterium?
}

\author{
Vera Flatz \\ Kerngebiet: Neuzeit \\ eingereicht bei: Ass.-Prof. Dr. Niels Grüne \\ eingereicht im: WiSe 2018/19 \\ Rubrik: Proseminar-Arbeit
}

\begin{abstract}
Societal and legal status of foreign language teachers in the early modern age. Language skills as the determining criterion?

The following paper deals with the requirements foreign language teachers had to fulfil in order to be granted citizenship in the Early Modern Age. The group of foreign language teachers was extremely heterogenous, originating from different countries, different social standings and lacking institutionalized education. Due to increased mobility and financial insecurities, foreign language teachers were rarely granted civil rights. This paper examines accepted and rejected requests for citizenship to explore what factors led to a positive or negative decision. As will be shown, multiple factors played a role in this process, including the language skills, but also factors such as prominent supporters and financial security.
\end{abstract}

\section{Einleitung}

Eine moderne Fremdsprache zu lernen ist heute Teil jeder fundierten Bildung. An jeder Bildungseinrichtung sind Stellen für Sprachlehrpersonen eingerichtet. Aus diesem Grund kommt Fremdsprachenlehrpersonen auch ein relativ hohes Maß an gesellschaftlicher Anerkennung entgegen, das anderen Fächern häufig fehlt. In der Frühen Neuzeit zeichnet sich ein ganz anderes Bild ab. 
Diese Arbeit untersucht, welches Maß an gesellschaftlicher Anerkennung, mit besonderem Blick auf den rechtlichen Status, Sprachmeister*innen ${ }^{1}$ im Laufe der Frühen Neuzeit entgegengebracht wurde und welche Faktoren einen bestimmten rechtlichen Status begünstigten. Der rechtliche Status war in der frühneuzeitlichen Stadt ein wichtiges Kriterium für die soziale Stellung eines Individuums. Dabei stand das Bürgerrecht für die volle Aufnahme in die Stadt. Neben diesem gab es auch die Möglichkeit, einen Beisitz oder Stadtschutz zu bekommen, um so in einer der beiden Reichsstädte Augsburg oder Nürnberg als Sprachmeister*in tätig sein zu können. ${ }^{2}$ Die folgende Untersuchung konzentriert sich auf diese beiden süddeutschen Reichsstädte im 17. und 18. Jahrhundert, da sie seit dem 15. Jahrhundert als wirtschaftliche, aber auch als kulturelle Zentren fungierten. ${ }^{3}$ Sprachmeister*in war während der gesamten Spanne der Frühen Neuzeit ein Beruf, der wenig gesellschaftliche Anerkennung erhielt, wodurch sich Sprachmeister*innen oft in einer prekären finanziellen Lage befanden. Es gab dennoch Sprachmeister*innen, die das Bürgerrecht oder zumindest einen Beisitz oder Stadtschutz verliehen bekamen. Die Gründe, aus denen Ansuchen angenommen oder abgelehnt wurden, waren vielfältig und hatten in manchen Fällen nichts mit der Nachfrage nach Sprachmeister*innen oder dem Können der Ansuchenden zu tun. Vielmehr spielten auch Faktoren wie die finanzielle Lage oder die Unterstützung lokaler Kaufleute eine Rolle.

Um diese These zu unterstützen, werden verschiedene, sowohl positiv als auch negativ ausgegangene Ansuchen beleuchtet, darunter der Beisitzantrag von Carl Peter Feretté in Augsburg, das Aufnahmegesuch von Johann Nicolaus Textor in Nürnberg und das Aufnahmegesuch von Nicolaus Grey in Augsburg. Als weiteres Beispiel für einen hochangesehenen Sprachmeister, der sogar Mitglied der Preußischen Akademien der Wissenschaften war, soll Matthias Kramer dienen. Neben der rechtlichen Situation sollen auch kurz die soziale Herkunft, der Grad der Ausbildung und die verschiedenen Anstellungsverhältnisse beleuchtet werden, um ein ganzheitlicheres Bild zu erhalten. Neben den genannten Quellen gibt es einige Werke in der Forschungsliteratur, die sich mit dem Thema der Sprachmeister*innen in der Frühen Neuzeit beschäftigen. Herausragend dabei sind die Werke von Mark Häberlein, der Herausgeber von mehreren Bänden zu diesem Thema ist. Einer dieser Bände ${ }^{4}$ beschäftigt sich explizit mit der Sozial- und Kulturgeschichte des Berufsstandes. Daneben gilt die von Helmut Glück, Mark Häberlein und Konrad Schröder verfasste Monografie "Mehrsprachigkeit in der Frühen Neuzeit" ${ }^{\prime \prime}$ als ein Standardwerk zum Thema, das sich auch den Sprachmeister*innen widmet.

1 Drei Prozent der Sprachmeister*innen, die im Bio-bibliographischen Lexikon der Fremdsprachenlehrer des deutschsprachigen Raumes vermerkt sind, sind weiblich: Konrad Schröder, Fremdsprachenlehrerinnen der Frühen Neuzeit. Zur Physiognomie der frühen Stadien eines modernen Frauenberufes, in: Mark Häberlein (Hrsg.), Sprachmeister. Sozial- und Kulturgeschichte eines prekären Berufsstandes, Bamberg 2015, S. 19-61, hier S. 24.

2 Helmut Glück/Mark Häberlein/Konrad Schröder, Mehrsprachigkeit in der Frühen Neuzeit. Die Reichsstädte Augsburg und Nürnberg vom 15. bis ins frühe 19. Jahrhundert, Wiesbaden 2013, S. 170.

3 Ebd., S. 9.

4 Mark Häberlein (Hrsg.), Sprachmeister. Sozial- und Kulturgeschichte eines prekären Berufsstands, Bamberg 2015.

5 Glück/Häberlein/Schröder, Mehrsprachigkeit in der Frühen Neuzeit. 


\section{Der Beruf der Sprachmeister*innen}

Im Laufe der Frühen Neuzeit gab es mehrere Möglichkeiten, eine Fremdsprache zu erlernen. Einerseits konnte eine Auslandsreise unternommen werden, um die Sprache des Landes in einem authentischen Umfeld und von Muttersprachler*innen zu lernen. Besonders Kaufleute schickten ihre Kinder auf Reisen, um deren fremdsprachliche Kompetenzen zu erweitern. Bei jungen Adeligen war die sogenannte "Grand Tour" im 17. und 18. Jahrhundert besonders beliebt, um die prestigeträchtigen Fremdsprachen vermittelt zu bekommen. ${ }^{6}$ Auf einer "Grand Tour" besuchten die jungen Adeligen, in Begleitung von Hofmeistern und anderen Bediensteten, die Höfe befreundeter Fürsten, aber auch Universitäten und Ritterakademien, um ihr standesgemäßes Verhalten zu vervollkommnen und Kenntnisse zu erwerben - darunter eben auch Fremdsprachen.? Außerdem gab es auch die Möglichkeit, im eigenen Land durch institutionalisierten Unterricht oder durch Privatunterricht Kenntnisse aufzubauen. Der Privatunterricht nahm hier jedoch die weitaus wichtigere Stellung ein, da es erst am Ende des 17. Jahrhunderts erste öffentliche Schulen gab, die neben Latein auch moderne Fremdsprachen in ihren Unterricht aufnahmen. Aus diesem Grund wurden moderne Fremdsprachen in der Frühen Neuzeit vorrangig von privaten Sprachmeister*innen gelehrt. ${ }^{8}$

\subsection{Herkunft}

Die Herkunftsländer der Sprachmeister*innen in den beiden Reichsstädten Augsburg und Nürnberg waren vielfältig. Dennoch kann in beiden Städten eine Dominanz der Sprachmeister*innen aus Frankreich festgestellt werden. Diese Dominanz lässt sich damit erklären, dass einerseits Französisch die wichtigste Fremdsprache der damaligen Zeit war. Andererseits strömten französische Muttersprachler*innen nach dem Widerruf des Ediktes von Nantes 1685 und nach der Französischen Revolution 1789 ins Heilige Römische Reich. Auch die konfessionelle Prägung der Städte spielte für die einwandernden Sprachmeister*innen eine Rolle: Die lutherische Stadt Nürnberg war für französische Katholik*innen nicht attraktiv. Französische Protestant*innen siedelten sich eher in der benachbarten Hugenottenstadt Erlangen an. Aus diesem Grund ist die Dominanz französischer Sprachmeister*innen in Augsburg noch ausgeprägter als in Nürnberg. Auch Italien spielte als Herkunftsland eine Rolle, in Nürnberg allerdings eine weit größere als in Augsburg. ${ }^{9}$

Als weitere Herkunftsländer sind die Schweiz, die südlichen Niederlande und Großbritannien zu nennen. In Nürnberg konstituierte sich die größte Gruppe jedoch nicht aus Muttersprachler*innen, sondern aus deutschsprachigen Sprachmeister*innen, welche die Fremdsprache ebenso nur als Zweitsprache beherrschten. Auch in Augsburg bildeten die Deutschsprachigen die zweitgrößte Gruppe. ${ }^{10}$ Ein Grund für die Dominanz der

6 Helmut Glück, Nürnberger Sprachmeister in der Frühen Neuzeit, in: Mark Häberlein/Christian Kuhn (Hrsg.), Fremde Sprachen in frühneuzeitlichen Städten, Wiesbaden 2010, S. 135-148, hier S. 135-138. Mathis Leibetseder, Die Kavalierstour. Adelige Erziehungsreisen im 17. und 18. Jahrhundert, Köln 2004, S. 9-10. Glück, Nürnberger Sprachmeister, S. 136. Glück/Häberlein/Schröder, Mehrsprachigkeit in der Frühen Neuzeit, S. 145-147. 
deutschsprachigen Sprachmeister*innen waren die Bedürfnisse der Kaufmannschaft, die sich neben fremdsprachlichen Kompetenzen auch eine Grundbildung im Kaufmännischen erwarteten. ${ }^{11}$ So vielfältig wie das Herkunftsland war auch der Stand, aus dem die Sprachmeister*innen kamen. Die Gruppe beinhaltete Glaubensflüchtlinge, besonders aus Frankreich, ebenso wie abgedankte Offiziere und Soldaten. Daneben waren auch ehemalige Kleriker, verarmte Adelige, Mediziner und Juristen, aber auch Handwerker in dieser Gruppe enthalten. Was viele von ihnen einte, waren die Brüche in ihrer Biografie und die hohe geografische Mobilität. ${ }^{12}$

\subsection{Ausbildung}

Bei der Frage nach der Ausbildung kann kein einheitliches Bild gezeichnet werden. Die Qualifikationen waren so vielfältig wie die Herkunft der Sprachmeister*innen. Es gab keine formelle, allgemein geregelte Ausbildung. Ebenso gab es auch keine institutionelle Zusammenarbeit in Gilden oder Zünften. Das bedeutet, dass der Beruf ein freies Gewerbe war, das nur über die Beschlüsse des Stadtrats geregelt wurde. Dieser entschied, wie vielen und welchen Sprachmeister*innen ein Aufenthalt genehmigt wurde. Außerdem konnte er in dieser Weise über das Angebot der Fremdsprachen in der entsprechenden Stadt entscheiden. ${ }^{13}$ Martin Zürn weist darauf hin, dass sich durch das in keiner Weise geregelte Gewerbe auch nichtqualifizierte Stümper unter den Sprachmeister*innen befanden. ${ }^{14}$ Auch in dieser Arbeit wird deutlich, dass fundierte fremdsprachliche Kenntnisse nicht bei allen Sprachmeister*innen vorhanden waren. Dennoch zeigen die in dieser Arbeit untersuchten Quellen, dass ein gewisses Maß an Zweisprachigkeit durchaus vorhanden war.

\subsection{Anstellungsverhältnisse}

Neben den privat lehrenden Sprachmeister*innen gab es auch einige wenige, die in einem Anstellungsverhältnis ihren Lohn verdienten. Dabei zu nennen sind die Hofund Pagensprachmeister*innen, die für die Ausbildung der adeligen Kinder in den Fremdsprachen zuständig waren. Diese Gruppe der Sprachmeister*innen war im Allgemeinen gebildet und verfügte über fundierte Fremdsprachenkenntnisse. ${ }^{15}$ Im 17. Jahrhundert wurde das Erlernen von Fremdsprachen zu einem der zentralen Ziele in der Erziehung des höfischen Nachwuchses. ${ }^{16}$

Auch an der Universität war es möglich, ein Anstellungsverhältnis als Sprachmeister*in zu bekommen, allerdings wurde universitärer moderner Fremdsprachenunterricht nur sporadisch angeboten. Die Jesuiten, die den Hauptteil des Lehrkörpers ausmachten,

11 Martin Zürn, Unsichere Existenzen. Sprachmeister in Freiburg i.Br., Konstanz und Augsburg in der Frühen Neuzeit, in: Mark Häberlein/Christian Kuhn (Hrsg.), Fremde Sprachen in frühneuzeitlichen Städten, Wiesbaden 2010, S. 103-121, hier S. 116.

12 Häberlein, Sprachmeister, S. 11

13 Glück/Häberlein/Schröder, Mehrsprachigkeit in der Frühen Neuzeit, S. 137.

14 Zürn, Unsichere Existenzen, S. 118.

15 Glück/Häberlein/Schröder, Mehrsprachigkeit in der Frühen Neuzeit, S. 138-139.

16 Mark Häberlein/Christian Kuhn (Hrsg.), Fremde Sprachen in frühneuzeitlichen Städten, Wiesbaden 2010, S. 15. 
waren der Ansicht, dass moderne Fremdsprachen vom Erwerb der lateinischen Sprache abhalten würden, und waren deshalb gegenüber Sprachmeister*innen skeptisch eingestellt. Außerdem waren die Universitätssprachmeister*innen nicht Mitglied der akademischen Korporation und hatten kein geregeltes Gehalt. Sie waren dazu gezwungen, unter den Studierenden selbst nach Schülern zu suchen. ${ }^{17}$ Im Laufe des 18. Jahrhunderts wurden die modernen Fremdsprachen in die Lehrpläne der Gymnasien und Fachschulen aufgenommen und erweiterten somit den Arbeitsmarkt für Sprachmeister*innen deutlich. Allerdings blieb das Angebot immer größer als die Nachfrage und der Großteil der Sprachmeister*innen musste versuchen, mit privatem Fremdsprachenunterricht ein Auskommen zu finden. ${ }^{18}$

\section{Rechtlicher Status - Aufnahme ins Bürgerrecht, Beisitz, Stadtschutz}

Als wichtiges Kriterium für die soziale Stellung eines Berufsstandes ist der rechtliche Status zu nennen. Das Bürgerrecht verliehen zu bekommen, war schwer und nur für einen kleinen Teil der mitteleuropäischen Stadtbewohner*innen möglich. Das Bürgerrecht beinhaltete viele Rechte, wie etwa die Teilnahme am politischen Leben, aber auch einige Pflichten, wie zum Beispiel Steuerleistungen. Um das Bürgerrecht verliehen zu bekommen, musste ein Bewohner bestimmte Voraussetzungen erfüllen, z. B. über ein Mindestvermögen verfügen. ${ }^{19}$ Ebenso konnten Fremde nicht sofort um das Bürgerrecht ansuchen, sondern mussten erst einige Jahre in der betreffenden Stadt verbracht und sich Kontakte in dieser aufgebaut haben. Außerdem spielte auch die Religion in konfessionell homogenen Städten eine wichtige Rolle. Diese Voraussetzungen schlossen bereits viele Sprachmeister*innen vom Bürgerrecht aus. Besonders die hohe Mobilität von Sprachmeister*innen, die von Stadt zu Stadt reisten, um ein Einkommen zu erzielen, erlaubte es ihnen oft nicht, die geforderte Dauer in einer Stadt zu leben. Aus diesem Grund strebte eine Vielzahl von Sprachmeister*innen das Bürgerrecht nicht an. Auch das erforderliche Mindesteinkommen übertraf die finanziellen Möglichkeiten von Sprachmeister*innen oft um ein Vielfaches. ${ }^{20}$

Für Personen, die das Bürgerrecht nicht anstrebten oder es aus anderen Gründen nicht verliehen bekamen, gab es andere Möglichkeiten, um sich beruflich in einer Stadt aufzuhalten. Dabei gab es die Möglichkeit des minderen Bürgerrechts. In Augsburg wurde dies als Beisitz, in Nürnberg als Stadtschutz und zum Teil auch als Beisassen- oder Hintersassenstatus bezeichnet. Dieser Status war an die Entrichtung einer bestimmten Gebühr gebunden und lief nach einiger Zeit ab, nach der eine Ausreise oder ein erneutes Ansuchen erfolgen musste. Daneben gab es auch die Möglichkeit einer Aufenthaltsgenehmigung für eine bestimmte Zeit, die ebenso an eine Gebühr geknüpft war. Beson-

17 Mark Häberlein, Der Wissensmarkt für Fremdsprachen im frühneuzeitlichen Europa, in: Marian Füssel/Philip Knäble/Nina Elseman (Hrsg.), Wissen und Wirtschaft. Expertenkulturen und Märkte vom 13. bis 18. Jahrhundert, Göttingen 2017, S. 225-363, hier S. 345-347.

18 Ebd.

19 Glück/Häberlein/Schröder, Mehrsprachigkeit in der Frühen Neuzeit, S. 170.

$20 \mathrm{Ebd}$ 
ders für die Frühe Neuzeit stellen die Akten der Bürgerrechts- und Beisitzansuchen eine wichtige Quelle dar, durch die viele Sprachmeister*innen erstmals sichtbar wurden. ${ }^{21}$

\subsection{Der Beisitzantrag des Sprachmeisters Carl Peter Feretté in Augsburg (1762)}

Carl Peter Feretté stammte aus Lothringen und beantragte im März 1761 den Beisitz in der Reichsstadt Augsburg. Zuerst berichtete Feretté von seinen bisherigen Tätigkeiten, um sein Können als Sprachmeister darzulegen. Er nannte Französisch als seine Muttersprache und gab an, an der Universität Freiburg Deutsch gelernt zu haben. Außerdem erwähnte er die Tatsache, dass er bereits mehrfach darum gebeten wurde, französischen und italienischen Sprachunterricht zu erteilen. ${ }^{22}$

„Durch man[n]igfaltig wohlanständigen Umgang und Converstion mit vielen respectivè ansehn[lichen] und fürnehmen Persohnen hießiger Stadt, hat sich unverhofft gefüget, daß sich zerschiedenen an mich addressiert, um sich meiner Information zu bedienen, wie ich dan[n] die Ehre habe, des Tit: PI: Herrn Graffen von Bißingen Page, dan[n] in H[errn] Neußens, H[errn] Schielens, v $\mathrm{H}$ [errn] Scravers und andern Kauffman[n]s Häusern mehr, deren Kinder und Bediente im Franzöß[ischen] zu instruiren."23

Die Antwort der Augsburger Steuermeister fiel positiv aus, da das Angebot der bisher ansässigen Sprachmeister*innen zu gering war, um die große Nachfrage der Augsburger Kaufmannschaft zu decken. Aus diesem Grund wurde das Ansuchen auf zwei Jahre bewilligt. ${ }^{24}$ Allerdings verließ Feretté die Stadt bereits vor Ablauf dieser zwei Jahre wieder. $^{25}$

Interessant erscheint bei dieser Quelle, dass die sprachlichen Fähigkeiten von Carl Peter Feretté sowie die Nachfrage nach Französischunterricht die Hauptrolle bei der Vergabe des Beisitzstatus gespielt haben. Die Steuermeister begründeten ihre Vergabe mit dem zu geringen Angebot an Französischunterricht in Augsburg. Da Augsburg eine große Kaufmannschaft hatte und Französisch zu einer der führenden Handelssprachen zählte, ist die Entscheidung der Steuermeister als vernünftig zu bewerten. So konnte sichergestellt werden, dass die jungen Kaufmannssöhne eine fundierte Fremdsprachenbildung erhielten, bevor sie die Geschäfte übernahmen. Außerdem schien es von Vorteil zu sein, dass Ferreté die Namen der Herren nannte, die sich Unterricht von ihm wünschten. Die Tatsache, dass sich Ferreté die Unterstützung der wichtigen Kaufmänner in Augsburg bereits gesichert hatte, hatte die Entscheidung der Steuermeister mit hoher Wahrscheinlichkeit ebenfalls beeinflusst. Einerseits war so ein Einkommen des Sprachmeisters nahezu gesichert, andererseits hatte er prominente Unterstützer, die sich für ihn bei den Steuermeistern einsetzen hätten können. Auch erwähnte Feretté in

21 Glück/Häberlein/Schröder, Mehrsprachigkeit in der Frühen Neuzeit., S. 171.

22 Beisitzantrag des Sprachmeisters Carl Peter Feretté, zit. nach Glück/Häberlein/Schröder, Mehrsprachigkeit in der Frühen Neuzeit, S. 549

23 Ebd.

24 Ebd.

25 Glück/Häberlein/Schröder, Mehrsprachigkeit in der Frühen Neuzeit, S. 549. 
seinem Gesuch, dass er bereits freie Kost und ein freies Zimmer habe, was zeigte, dass er ernsthafte Absichten hegte, für längere Zeit in dieser Stadt zu verbleiben. ${ }^{26}$ Dieses Beispiel verdeutlicht, dass die sprachlichen Fertigkeiten und die tatsächliche Nachfrage eine Rolle bei der Vergabe des Beisitzstatus gespielt haben. Trotzdem werden bereits hier andere Faktoren, wie die Nennung prominenter Namen oder das Vorhandensein einer Unterkunft, sichtbar, die ebenso die Vergabe beeinflusst haben könnten.

\subsection{Das Aufnahmegesuch des Sprachmeisters Johann Nicolaus Textor in Nürnberg (1764-1766)}

Dieses Aufnahmegesuch gibt einen guten Einblick in die verschiedenen Stationen eines Aufnahmegesuches in Nürnberg. Johann Nicolaus Textor, der angab, aus Luxemburg zu stammen, ersuchte um die Aufnahme in den Stadtschutz. Er erklärte, Jura studiert zu haben, schon einige Jahre als französischer Sprachmeister tätig gewesen zu sein und bei verschiedenen Herren eine Anstellung gefunden zu haben. Außerdem gab er an, dass er bereits um die Stelle als Sprachmeister an einer städtischen Schule angesucht habe, inm diese Stelle aber verwehrt geblieben sei. Er bat daher um eine Aufnahme in den Stadtschutz, da ihm der Aufenthalt in der Stadt nicht länger gewährt wurde. $^{27}$

„Nachdeme mir nun der Aufenthalt alhier nicht länger gestattet werden will, so ergehet an Euer Hochwohlgeb[orene] Gnaden hiermit mein unterthänig höchstangelegenstes Bitten, mir den alhiesigen Stadt-Schuz aus Oberherrl[icher] hoher Gnade angedeyen zu lassen".28

Die darauffolgende Kommunikation zwischen dem Vormundamt und dem Stadtrat zeigt, was für Nachweise Textor erbringen musste, um den Stadtschutz zu erhalten. Textor wurde angewiesen, Proben seines Könnens vorzulegen, was sich allerdings mehrere Male verzögerte. Erst nach mehrmaligem Nachfragen des Vormundamtes gab es eine Textprobe von Textor. ${ }^{29}$

Besonders interessant an diesem Beispiel sind die Gründe, aufgrund derer Textors Stadtschutzgesuch schlussendlich bewilligt wurde. Bereits in seinem Gesuch gab er an, die geforderte Kaution von 25 Gulden hinterlegt zu haben. Diese Tatsache wurde auch später in einem Ratserlass positiv erwähnt. Außerdem unterzeichneten sein Ansuchen zwei Kaufmänner, die sich als Unterstützter Textors positionierten. Die erste Stellungnahme des Vormundamtes zeigt, dass es in der Stadt Nürnberg zu dieser Zeit keinen Mangel an Französischlehrer*innen gab. Die bereits bekannten Sprachmeister*innen beklagten sich sogar über zu wenig Arbeit und daraus folgend über zu wenig Lohn, um ein gutes Auskommen zu finden. Ebenso zeigt eine weitere Stellungnahme des Vormundamts, dass die geforderten Proben seiner Arbeit keinem hohen Standard ent-

26 Glück/Häberlein/Schröder, Mehrsprachigkeit in der Frühen Neuzeit, S. 549.

27 Aufnahmegesuch des Sprachmeisters Johann Nicolaus Textor in Nürnberg, zit. nach Glück/Häberlein/Schröder, Mehrsprachigkeit in der Frühen Neuzeit, S. 552. 
sprochen haben. Es wurden Grammatikfehler angemerkt, die zeigen, dass die sprachlichen Kompetenzen nicht für einen positiven Bescheid des Aufnahmegesuchs gereicht hätten: „Es ist einem Gelehrten dieser Sprache verständigen zur Beurtheilung gegeben worden, der es nicht gelobet, ja gar Grammatical[ische] Fehler angemerket, doch bleibet es bei dem Lob derer, wo er informiret."30

Das Beispiel des Johann Textors zeigt deutlich, dass neben den sprachlichen Kompetenzen und der Nachfrage nach Fremdsprachenunterricht in einer Stadt auch andere Faktoren wichtig für die Entscheidung waren, ob ein Stadtschutz vergeben wurde oder nicht. Obwohl das Vormundamt mehrere Gründe darlegte, die gegen eine Aufnahme gesprochen hätten (die fehlende Nachfrage, das Vorhandensein mehrerer Sprachmeister*innen und das schlechte Können), wurde Textor in den Stadtschutz aufgenommen. Hier müssen also andere Gründe den Ausschlag für einen positiven Bescheid gegeben haben. Die Kommunikation, die diesem Bescheid vorausgeht, zeigt deutlich, dass dies sowohl die hinterlegten 25 Gulden als auch die Unterstützung eingesessener Kaufleute waren. Im Bericht des Vormundamts wurde dargelegt, dass sich verschiedene Handelsleute für Textor ausgesprochen haben und sein Können bezeugten.

"Was seine informationes anbelanget, so hat er sich auf die Handelsleute Kißling, Söldel und Liebel bezogen, ausser welchen er verschiedene Handelsdiener informiere. Söldel und Liebel geben ihm auch auf Befragen ein sehr gutes Zeugnis einer Geschicklichkeit, so daß, wenn seine pronunciation ebenso gut wäre, deselbe vorzügl[ich] zu rühmen seyn müste, indeme die Unterweisung vortreflich, weswegen sie selbst inständig gebetten, ihm den hiesigen Aufenthalt zu erlauben".31

Diese Unterstützung scheint letztlich den Ausschlag gegeben zu haben, das Aufnahmegesuch positiv zu beurteilen.

Was in den besprochenen Beispielen keine Rolle spielte, ist die Religion des Ansuchenden. Beim Ansuchen von Textor gab es einen Vermerk, dass zu der Religionszugehörigkeit nichts bekannt sei. Ein Kommentar ergänzte, dass Textor römisch-katholischen Glaubens war. ${ }^{32}$ Dieser Umstand spielte aber in der weiteren Kommunikation keine Rolle mehr. Daraus lässt sich schließen, dass die Religionszugehörigkeit in diesem Beispiel nicht von Bedeutung war. Auch in den anderen Beispielen wurde die Religionszugehörigkeit nicht explizit genannt und floss nicht in den Entscheidungsprozess mit ein. Dies bedeutet jedoch nicht, dass eine falsche Religionszugehörigkeit in manchen Fällen kein Faktor war, der eine Ablehnung begünstigte.

\subsection{Das Aufnahmegesuch des Sprachmeisters Nicolaus Grey in Augsburg (1633)}

Dieses Aufnahmegesuch soll ein Beispiel für einen negativ ausgegangenen Fall darstellen. In den bereits besprochenen Beispielen wurden Gründe verschiedener Natur

30 Aufnahmegesuch des Sprachmeisters Johann Nicolaus Textor in Nürnberg, zit. nach Glück/Häberlein/Schröder, Mehrsprachigkeit in der Frühen Neuzeit, S. 552. 
dargelegt, die letztlich zu einem positiven Bescheid führten. Es gab jedoch auch eine Vielzahl an Gesuchen, die abgelehnt wurden. Hier stellt sich die Frage, ob die Kriterien für eine Ablehnung dieselben waren wie die Kriterien, aufgrund derer ein Gesuch angenommen wurde.

Der Sprachmeister Nicolaus Grey bat 1633 um die Erlaubnis, in Augsburg als Fremdsprachenlehrer tätig zu sein. Schon in Nürnberg war er als Sprachmeister beschäftigt gewesen und versuchte nun, als italienischer Sprachmeister in Augsburg ein Auskommen zu finden. Sein Ansuchen begründete er mit der Tatsache, dass in der Handelsstadt ein Bedarf an Italienischunterricht herrschte, der bisher nicht erfüllt werden konnte. ${ }^{33}$

Die Antwort der Verordneten zum Schulwesen fiel sehr kritisch aus. Sie äußerten sich zu der Gesamtentwicklung des Augsburger Schulsystems, das sich mehr auf die deutsche und die lateinische Sprache konzentrieren wollte. Als moderne Fremdsprache wurde neben den genannten beiden lediglich Französisch akzeptiert. Die Zuständigen gaben zwar an, ein Interesse an der Gründung einer italienischen Schule in der Stadt zu haben, kritisierten aber, dass die Schüler aufgrund eines Überangebots an Sprachen überfordert sein könnten. Einerseits wurde befürchtet, dass durch eine zusätzliche italienische Schule einige Eltern ihre Kinder nicht mehr in den Lateinunterricht schicken würden. Andererseits wurde ebenso das gleichzeitige Lernen von mehr als einer modernen Fremdsprache abgelehnt. ${ }^{34}$

„Od[er] doch beede sprachen neben einander lehrnen wolten lassen, da es doch vnmöglich vnd gantz wider vnsern methotum, auch die vernunfft selbsten, $d z$ man zwo Sprachen mit vnd neben einander vnd also auf einmahl ergreiffe [...] vnd eben diß ist bishero neben anderen erroribus nicht der geringste gewesen, $d z$ man inn Schulen zu einer Zeit vilerley sachen tractiert, vnd damit die zarte Jugendt verwirret hat".35

Dieses Beispiel zeigt deutlich, dass es neben den unzureichenden fremdsprachlichen Kompetenzen der Sprachmeister*innen auch andere Gründe für die Ablehnung eines Ansuchens gab. In diesem Fall spielte der Fokus im Schulwesen eine entscheidende Rolle. Obwohl das Erlernen von Italienisch, einer Handelssprache, Vorteile für junge Kaufmannssöhne gebracht hätte, wollten diejenigen, die für das Schulwesen verantwortlich waren, keine weitere moderne Fremdsprache in ihr Schulwesen integrieren. Hier wird die Vorherrschaft des Lateinischen gut sichtbar. Aus Angst, dass einige Eltern ihre Kinder dann nur noch in den Französisch- oder den Italienischunterricht schicken würden, wurde eine weitere Fremdsprache aus Prinzip abgelehnt. Das Argument, dass mehrere Sprachen nicht zur gleichen Zeit gelernt werden können und sogar zu einer verminderten kognitiven Leistung führen, entbehrt zwar nach heutigen wissenschaftlichen Erkenntnissen jeglicher Grundlage, war aber bis ins 20. Jahrhundert weit verbreitet. ${ }^{36}$

33 Aufnahmegesuch des Sprachmeisters Nicolaus Grey in Augsburg, zit. nach Glück/Häberlein/Schröder, Mehrsprachigkeit in der Frühen Neuzeit, S. 484.

34 Ebd.

35 Ebd., S. 485.

36 Michael Paradowski/Aleksandra Bator, Perceived Effectiveness of Language Acquisition in the Process of Multilingual Upbringing by Parents of Different Nationalities, in: International Journal of Bilingual Education and 
Die Vorherrschaft des Lateinischen als Sprache der katholischen Kirche und als Sprache der Gelehrten blieb bis ins 18. Jahrhundert hinein erhalten. ${ }^{37}$ Französisch war als Modesprache der Zeit lange die einzige moderne Fremdsprache, welche in nennenswertem Umfang unterrichtet wurde. Dies wird auch durch die Herkunft der Fremdsprachenlehrer bestätigt. Obwohl Italienisch neben Französisch die wichtigste Sprache für Augsburg war, kamen nur 7 Prozent der Sprachmeister*innen aus Italien. Dieser Anteil war deutlich geringer als jener der italienischsprachigen Lehrer*innen in Nürnberg. ${ }^{38}$ Auch der Angebotsanteil an Italienisch war mit lediglich 22 Prozent deutlich geringer als jener in Nürnberg. Die Stellungnahme der Verordneten zum Schulwesen zeigt einen Grund auf, weshalb das Italienische in Augsburg im Nachteil war. Durch die Entscheidungen, italienischsprachigen Sprachmeister*innen den Aufenthalt zu verwehren, aus Angst, die Dominanz des Lateinischen zu gefährden, blieb das Angebot an Italienischunterricht relativ gering.

\section{Matthias Kramer als herausragendes Beispiel}

Matthias Kramer, 1640 in Köln geboren, Sprachmeister, Didaktiker und Grammatiker, gilt als einer der herausragendsten Sprachmeister*innen der Frühen Neuzeit. Keine/r seiner zeitgenössischen Kollegen*innen produzierte ein so umfangreiches Gesamtwerk wie er, bestehend aus Grammatiken, Dialogsammlungen und didaktischen Abhandlungen. Dieses Kapitel wird diese herausragende Figur etwas näher beleuchten. Abschließend soll festgestellt werden, welchen rechtlichen Status er sich schlussendlich erarbeiten konnte.

Obwohl Kramer einer der produktivsten Sprachmeister*innen seiner Zeit war, viele hochangesehene Schüler*innen hatte und in die Königlich Preußische Societät der Wissenschaft aufgenommen wurde, ${ }^{39}$ war sein Leben geprägt von finanzieller Unsicherheit und fehlender sozialer Anerkennung. ${ }^{40} 1669$ bat Kramer in der Stadt Nürnberg um den Schutzverwandtenstatus. Dieser wurde inm genehmigt und viele Jahre um jeweils ein weiteres Jahr verlängert. ${ }^{41}$ Dennoch wurde ihm zeitlebens das Nürnberger Bürgerrecht verwehrt. Seine materielle Not war am Ende seines Lebens so groß, dass er sich im Alter von achtzig Jahren dazu entschloss, nach Erlangen zu übersiedeln, da er sich dort durch eine Anstellung an der Ritterakademie ein sichereres Einkommen erhoffte. ${ }^{42}$ Obwohl er in Erlangen eine Fixanstellung hatte, suchte er ein Jahr nach der Übersiedelung um eine Pension an, da seine Einkünfte trotz der Stelle gering blieben.

Bilingualism 21 (2018), Heft 6, S. 647-665, hier S. 650.

37 Häberlein, Fremde Sprachen in Frühneuzeitlichen Städten, S. 11.

38 Glück/Häberlein/Schröder, Mehrsprachigkeit in der Frühen Neuzeit, S. 145-148.

39 Die Forschungsfelder der Königlich Preußischen Societät waren für diese Zeit ungewöhnlich breit gefächert. Auch geisteswissenschaftliche Forschung im Bereich der Sprachwissenschaften wurde betrieben: Simon Kofler, Akademiegründungen im Geiste der "New Science”. Royal Académia des Sciences und Berliner Sozietät im Vergleich, Dipl. Innsbruck 2018, S. 40.

40 Häberlein, Sprachmeister, S. 9

41 Glück/Häberlein/Schröder, Mehrsprachigkeit in der Frühen Neuzeit, S. 155.

42 Häberlein, Sprachmeister, S. 9-10. 
Ob dieses Unterfangen erfolgreich war, lässt sich nicht mehr beurteilen. Noch im selben Jahr verstarb Matthias Kramer. ${ }^{43}$

Kramer selbst reflektierte in einigen seiner Werke über seine eigene Situation und die Situation von Sprachmeister*innen seiner Zeit im Allgemeinen. Er tat das entweder explizit in Vorwörtern zu seinen Werken oder verpackte seine Meinung implizit in die didaktischen Dialoge seiner Lehrwerke. Im Vorwort eines seiner Werke beklagte er sich über seine finanzielle Situation, indem er sich als mittellosen, dienstlosen und wohnungslosen Mann bezeichnete. In einem seiner didaktischen Dialoge unterhalten sich Schüler und Meister darüber, dass nicht einmal das Schreiben von Büchern genug Geld einbringe. Andere Dialoge enthalten Szenen, die im Leben der Sprachmeister*innen mit Sicherheit keine Realität waren und können so als eine Art Wunschdenken Kramers interpretiert werden.4 "Dann wird die Zwiespältigkeit des Lebens deutlich, das französische Sprachmeister im 17. Jahrhundert führen mußten. Stets die Welt des Luxus vor Augen habend, für kurze Momente daran teilhabend, mußten sie doch gleichzeitig oft um ihre Existenz bangen. ${ }^{\prime 45}$

Das Beispiel Kramers zeigt, dass selbst die herausragendsten und berühmtesten Sprachmeister*innen ihrer Zeit oft nicht dieselbe rechtliche und auch soziale Stellung hatten wie Mitglieder anderer Berufsgruppen. Obwohl Kramer einen Stadtschutzstatus hatte, musste dieser jedes Jahr erneuert werden, was an eine Gebühr geknüpft war. Zeitlebens blieb inm die Anerkennung als Bürger der Stadt Nürnberg verwehrt. Nur wenigen Sprachmeister*innen gelang es, materielle und finanzielle Sicherheit aufzubauen, indem sie das Bürgerrecht in einer Stadt erlangten. ${ }^{46}$

\section{Resümee}

Sprachmeister*innen in der Frühen Neuzeit waren eine sehr heterogene Gruppe, die sich in ihrer Herkunft, ihrem Ausbildungsgrad und in ihren Anstellungsverhältnissen unterschieden. Dennoch einte sie die Tatsache, dass das Leben als Sprachmeister*in finanzielle Schwierigkeiten, eine hohe Mobilität und eine niedrige soziale Stellung mit sich brachte. Das Bürgerrecht einer Stadt zu erhalten war die Ausnahme und blieb sogar dem berühmten Matthias Kramer Zeit seines Lebens verwehrt. Als Alternative blieb das mindere Bürgerrecht, das auch Sprachmeister*innen immer wieder zugesprochen bekamen.

Carl Peter Ferettés Beisitzantrag zeigt, dass fundierte Fremdsprachenkenntnisse und die Nachfrage nach einer bestimmten Sprache wichtige Gründe waren, aus denen ein Beisitz oder Stadtschutzstatus vergeben wurde, allerdings waren diese Faktoren nicht

43 Glück/Häberlein/Schröder, Mehrsprachigkeit in der Frühen Neuzeit, S. 156.

44 Harald Völker, Matthias Kramer als Sprachmeister, Didaktiker und Grammatiker für die französische Sprache in Deutschland, in: Wolfgang Dahmen u. a. (Hrsg.), "Gebrauchsgrammatik“ und "Gelehrte Grammatik”. Französische Sprachlehre und Grammatikographie zwischen Maas und Rhein vom 16. bis zum 19. Jahrhundert, Tübingen 2001, S. 167-250, hier S. 210-211. 
immer die ausschlaggebenden. Das Aufnahmegesuch von Johann Nicolaus Textor zeigt deutlich, dass seine eher mangelhaften Fremdsprachenkenntnisse nicht der wesentliche Faktor für die Entscheidung gewesen sein können. Auch die Tatsache, dass der Markt an Französischlehrpersonen in Nürnberg zu diesem Zeitpunkt bereits gesättigt war, beeinflusste Textors Ansuchen nicht zum Negativen. Ausschlaggebend waren oft die finanziellen Möglichkeiten, welche die Sprachmeister*innen hatten, um jährlich die geforderte Gebühr zu bezahlen. Außerdem war auch prominente Unterstützung in einer Stadt eine wichtige Grundlage für ein erfolgreiches Ansuchen. Beide Punkte werden anhand von Textors Ansuchen deutlich. Die Hinterlegung von 25 Gulden und die Unterstützung prominenter Handelsleute hatte wohl den Ausschlag zu einer Aufnahme in den Stadtschutz gegeben. Daneben hat sich gezeigt, dass nicht nur die Person der Sprachmeister*innen an sich, sondern auch die angebotene Sprache eine Rolle spielte. Waren in einer Stadt schon viele Fremdsprachenlehrpersonen einer bestimmten Sprache vertreten, wollten die Verantwortlichen nicht eine weitere Fremdsprache, die daneben noch mit Latein konkurrieren hätte können. Dies wird anhand des Ansuchens des italienischen Sprachmeisters Nicolaus Grey deutlich. Seine Fremdsprachenkenntnisse werden in der Begründung der Ablehnung gar nicht erwähnt, sondern die allgemeine Fokussierung des Bildungssystems auf das Französische war entscheidend. Auch wenn diese Betrachtung mit ihrer geringen Fallzahl keine allgemeingültige Aussagekraft hat, zeigt sich dennoch, dass auf keinen Fall nur das sprachliche Können und die Nachfrage in einer Stadt entscheidend für den Ausgang eines Ansuchens waren. Die Gründe für einen positiven oder negativen Bescheid waren vielfältig und lassen sich nur fallweise betrachten.

\section{Literatur}

Glück, Helmut, Nürnberger Sprachmeister in der Frühen Neuzeit, in: Mark Häberlein/ Christian Kuhn (Hrsg.), Fremde Sprachen in frühneuzeitlichen Städten, Wiesbaden 2010, S. 135-148.

Ders./Häberlein, Mark/Schröder, Konrad, Mehrsprachigkeit in der Frühen Neuzeit. Die Reichsstädte Augsburg und Nürnberg vom 15. bis ins frühe 19. Jahrhundert, Wiesbaden 2013.

Häberlein, Mark (Hrsg.), Sprachmeister. Sozial- und Kulturgeschichte eines prekären Berufsstands, Bamberg 2015.

Ders., Der Wissensmarkt für Fremdsprachen im frühneuzeitlichen Europa, in: Marian Füssel/Philip Knäble/Nina Elseman (Hrsg.), Wissen und Wirtschaft. Expertenkulturen und Märkte vom 13. bis 18. Jahrhundert, Göttingen 2017, S. 225-363.

Ders./Kuhn, Christian (Hrsg.), Fremde Sprachen in frühneuzeitlichen Städten, Wiesbaden 2010.

Kofler, Simon, Akademiegründungen im Geiste der "New Science“. Royal Académia des Sciences und Berliner Sozietät im Vergleich, Dipl. Innsbruck 2018. 
Leibetseder, Mathis, Die Kavalierstour. Adelige Erziehungsreisen im 17. und 18. Jahrhundert, Köln 2004.

Paradowski, Michael/Bator, Aleksandra, Perceived Effectiveness of Language Acquisition in the Process of Multilingual Upbringing by Parents of Different Nationalities, in: International Journal of Bilingual Education and Bilingualism 21 (2018), Heft 6, S. 647-665.

Schröder, Konrad, Fremdsprachenlehrerinnen der Frühen Neuzeit. Zur Physiognomie der frühen Stadien eines modernen Frauenberufes, in: Mark Häberlein (Hrsg.), Sprachmeister. Sozial- und Kulturgeschichte eines prekären Berufsstandes, Bamberg 2015, S. $19-61$.

Völker, Harald, Matthias Kramer als Sprachmeister, Didaktiker und Grammatiker für die französische Sprache in Deutschland, in: Wolfgang Dahmen u. a. (Hrsg.) " "Gebrauchsgrammatik" und "Gelehrte Grammatik". Französische Sprachlehre und Grammatikographie zwischen Maas und Rhein vom 16. bis zum 19. Jahrhundert, Tübingen 2001, S. $167-250$.

Zürn, Martin, Unsichere Existenzen. Sprachmeister in Freiburg i.Br., Konstanz und Augsburg in der Frühen Neuzeit, in: Mark Häberlein/Christian Kuhn (Hrsg.) Fremde Sprachen in frühneuzeitlichen Städten, Wiesbaden 2010, S. 103-121.

Vera Flatz ist Masterstudentin im Masterstudiengang Lehramt für Sekundarstufe für die Fächer Englisch und Geschichte, Sozialkunde \& Politische Bildung an der Universität Innsbruck. vera.flatz@student.uibk.ac.at

\section{Zitation dieses Beitrages}

Vera Flatz, Gesellschaftlicher und rechtlicher Status der Sprachmeister*innen in der Frühen Neuzeit. Sprachliche Fähigkeiten als entscheidendes Kriterium?, in: historia. scribere 12 (2020), S. 207-219, [http://historia.scribere.at], eingesehen 15.6.2020 (=aktuelles Datum).

Creative Commons Licences 3.0 Österreich unter Wahrung der Urheberrechte der Autorlnnen. 
